\title{
Sex-specific effect of leptin on gene expression in placentas and fetal tissues in mice
}

\author{
E. Denisova*, E. Makarova \\ Institute of Cytology and Genetics SB RAS, Novosibirsk, Russia \\ *e-mail:elena_nsib@list.ru
}

Key words: leptin, placenta, fetal brain, pregnancy, gene expression, mice

Aim: According to the DOHAD hypothesis, the health of individuals depends on the conditions of prenatal development [1]. The hormone leptin is elevated in obese animals. Hyperleptinemia during pregnancy was shown to affect the metabolic phenotype of the offspring, and this effect may depend on the offspring sex [2]. Leptin receptors were found in the placenta and fetal brain and liver. Programming leptin effects may be mediated via leptin influence on the placental functions and development of fetuses. The goal of the work was to examine how leptin administration to the mice at the end of pregnancy affects expression of genes encoding signal and transport proteins in placentas and genes involved in energy homeostasis regulation in the brain of male and female fetuses.

Methods: Leptin or saline (control) were administered to C57B1 mice at the day 17 of pregnancy. Weight of fetuses and placentas, relative expression of genes encoding IGF1, IGF2 and IGF2R, glucose transporters GLUT1 and GLUT3, amino acid transporters SNAT1, SNAT2 and SNAT4 in placentas, IGF1, IGF2 and IGF2R in liver, and MCR4, AgRP, NPY and POMC in brain were measured in male and female fetuses within 3 and 7 hours after injection. Leptin plasma concentrations were measured in fetuses and pregnant females within $1 \mathrm{~h}$ after injection.

Results: Through $1 \mathrm{~h}$ after leptin administration, leptin concentrations in fetal and mother blood were significantly higher than in saline treated mice. Through $3 \mathrm{~h}$ after leptin administration, gene expression was changed only in female foetuses: the expression of IGF2 was decreased in female placentas and expression of MCR4 was increased in fetal female brain. Through $7 \mathrm{~h}$ after leptin administration, IGF2R gene expression was increased in liver of female fetuses, AgRP gene expression was decreased in brain of male fetuses, MCR4 gene expression in brain was increased and fetal weight was decreased in fetuses of both sexes.

Conclusion: Leptin administration retards fetal growth rate via the sex-specific molecular mechanisms. In female fetuses, these mechanisms include inhibition of IGF2 expression in placentas and activation of IGF2R expression in liver. In male and female fetuses, leptin administration differently affects the expression of genes that regulate energy homeostasis at maturity. Gender-specific programming effect of maternal leptin may be associated with different gene expression response to leptin in placentas, brain and liver of male and female fetuses.

Acknowledgements: Supported by the RFBR (17-04-01357A and 14-04-00694A).

\section{References}

1. Wadhwa P.D. et al. (2009) Developmental origins of health and disease: brief history of the approach and current focus on epigenetic mechanisms. Semin Reprod Med. 27(5):358-368.

2. Makarova E.N. et al. (2013) Influence of abnormally high leptin levels during pregnancy on metabolic phenotypes in progeny mice. Am J Physiol Regul Integr Comp Physiol. 305:R1268-R1280. 